This item was submitted to Loughborough's Research Repository by the author.

Items in Figshare are protected by copyright, with all rights reserved, unless otherwise indicated.

\title{
Trapped modes in the water-wave problem for a freely-floating structure
}

PLEASE CITE THE PUBLISHED VERSION

LICENCE

CC BY-NC-ND 4.0

\section{REPOSITORY RECORD}

Mclver, P., and M. Mclver. 2019. "Trapped Modes in the Water-wave Problem for a Freely-floating Structure". figshare. https://hdl.handle.net/2134/476. 


\title{
Trapped modes in the water-wave problem for a freely-floating structure
}

\author{
P. McIver and M. McIver \\ Department of Mathematical Sciences, Loughborough University, \\ Loughborough, Leics, LE11 3TU, UK
}

\begin{abstract}
Trapped modes in the linearized water-wave problem are free oscillations of an unbounded fluid with a free surface that have finite energy; it has been known for some time that such modes are supported by certain structures when held fixed. This paper investigates the problem of a freelyfloating structure that is able to move in response to the hydrodynamic forces acting upon it and it is shown that trapped modes also exist in this problem. For a freely-floating structure a trapped mode is a coupled oscillation of the fluid and the structure.
\end{abstract}

\section{Introduction}

It is known that, within the linearized theory of water waves, certain structures when held fixed can support a trapped mode of a particular frequency (McIver 1996). Such a mode is a free oscillation of an unbounded fluid with a free surface that has finite energy, does not radiate waves to infinity, and in the absence of viscosity will persist for all time. If, for a specified frequency of fluid oscillation, the structure does not support a trapped mode, then the solutions to the frequency-domain radiation and scattering problems at that frequency are unique. From the practical point of view, the existence of a trapped mode means that it is difficult to find accurate numerical solutions to the radiation and scattering problems for a range of frequencies around the trapped-mode frequency (Newman 1999).

McIver (1997) showed that trapped modes are orthogonal to any incident wave and consequently will not be excited in a scattering problem in either the time or frequency domains. However, the existence of a trapped mode implies that at the trapped-mode frequency there is a pole in a frequency-domain radiation potential and the solution to the corresponding radiation problem does not exist at that frequency. A consequence of this is that trapped modes can be excited in the time domain by the forced oscillations of a trapping structure (McIver, McIver \& Zhang 2003).

Recently, it has been established that the trapped modes supported by a fixed structure, as described above, cannot be excited when that structure is allowed to float freely (and hence respond to the hydrodynamic forces on it), with or without incident waves (McIver 2005). For motion 
in a single mode this follows immediately from the frequency-domain equation of motion which shows that the pole in the radiation potential at the trapped-mode frequency is annulled by a corresponding zero in the velocity. However, it was also found that trapped modes supported by fixed structures cannot be excited by more general motions of the structure that involve more than one mode of oscillation. Thus, although the existence of such trapped modes leads to numerical difficulties in the solution of the frequency-domain radiation and scattering problems, their existence has no direct relevance to the problem of a freely-floating body in which the radiation and scattering problems are combined through the equation of motion. It is therefore natural to investigate questions of uniqueness, and in particular the existence of trapped modes, in the problem of a freely-floating structure.

Remarkably, there appears to be only one published result on uniqueness in the problem for a freely-floating structure and that is due to John (1950) (although Kuznetsov, 2003, has applied the ideas in the proof to a structure supported by an air cushion). Suppose a structure satisfies the conditions that vertical lines drawn downwards from the free surface do not intersect the structure and that no part of the free surface is isolated from infinity, then John's result states that there is a unique solution to the problem of a freely-floating structure, and hence no trapped modes, provided that the frequency is sufficiently high.

The purpose of the present work is to demonstrate the existence of freely-floating structures that do support trapped modes; these modes correspond to coupled free oscillations of both the structure and the surrounding fluid. In line with the terminologies employed by McIver (2005) in the context of a more general discussion of resonances, fixed structures that support persistent free oscillations of the fluid will be called "sloshing trapping structures", while structures that may oscillate freely in the presence of the fluid will be called "motion trapping structures". The method used in the constructions described here is an inverse procedure similar to that used by Kyozuka and Yoshida (1981) to obtain wave-free oscillating structures, and by McIver (1996) to obtain sloshing trapping structures. All of the examples presented violate both of the conditions in John's theorem and, in particular, there is always a portion of the free surface that is isolated from infinity. John's result does not rule out trapped modes for structures that have no isolated portion of the free surface, but attempts to find such structures have failed.

For a structure to support motion trapped modes of a particular frequency $\omega=\omega_{0}$ in a single mode of oscillation, the corresponding frequency-dependent hydrodynamic coefficients must satisfy two conditions at this frequency. First of all the damping coefficient must be zero, so that there is no wave radiation to infinity, and secondly the added mass coefficient must satisfy a resonance condition obtained from the requirement that the homogeneous form of the equation of motion for the structure has a non-trivial solution. The latter condition may be rewritten in terms of properties of the velocity potential by an application of Green's theorem. Trapping structures are found by an application of the inverse procedure that uses a singular velocity potential that does not radiate waves to infinity and satisfies the additional requirements imposed by the new form of the resonance condition. Motion trapping structures are then obtained by examination of the streamlines corresponding to a modified potential that ensures that the correct boundary condition 
on the surface of the structure is satisfied. Unlike sloshing trapping structures, there is no singular behaviour at the trapped-mode frequency in any of the hydrodynamic coefficients.

This paper is concerned with two-dimensional motions and attention is focussed mainly on structures that are constrained to move in heave. The problem is formulated in $\S 2$ and the modified form of the resonance condition is obtained in $\S 3$. Specific constructions of motion trapping structures are described in $\S 4$ and some consequences of the existence of motion trapping structures are discussed in $\S 5$. Finally, in $\S 6$, it is demonstrated that the restriction to vertical motions is not necessary for the existence of motion trapping structures and an example that oscillates horizontally is described briefly.

\section{Formulation}

Attention is restricted to motion in two dimensions and Cartesian coordinates $x, z$ are chosen with $z$ directed vertically upwards from the mean free surface. In addition, plane polar coordinates $R, \theta$ will be used where $\theta$ is measured from the downward vertical so that $z=-R \cos \theta$.

Consider a moored surface-piercing structure constrained to move in the vertical direction with

initial displacement $Z(0)$ and initial velocity $\dot{Z}(0)$. The Fourier transform of the time-domain equation of motion yields the frequency-domain equation of motion

$$
\left[\rho g W+\kappa-\omega^{2}\{M+a(\omega)+\mathrm{i}(b(\omega)+\gamma) / \omega\}\right] v(\omega)=-\mathrm{i} \omega[X(\omega)+M \dot{Z}(0)]-(\rho g W+\kappa) Z(0)
$$

(see, for example, McIver 2005; the Fourier transform is defined so that the frequency-domain motions are equivalent to oscillations with a time dependence $\left.\mathrm{e}^{-\mathrm{i} \omega t}\right)$. Here $\rho$ is the fluid density, $g$ is the acceleration due to gravity, $W$ is the water plane area, $\kappa$ is the spring constant of the moorings, $M$ is the mass of the structure (which by Archimedes principle is $\rho$ times the submerged volume $V), a$ is the added mass coefficient, $b$ is the damping coefficient, $\gamma$ is the damping constant of the moorings, $v$ is the (complex) amplitude of the structural velocity, and $X$ is the exciting force due to wave incidence on a fixed structure. Note that the functions of frequency in equation (1) are Fourier transforms in time of physical quantities so that, for example, $v(\omega)$ has the dimensions of length rather than velocity. The inclusion in $(1)$ of $Z(0)$ and $\dot{Z}(0)$ is significant in considerations of the behaviour in the time domain of motion trapping structures.

\section{Conditions for heave resonance}

Investigation of the uniqueness of the solution to a problem involving a freely-floating structure in heave leads to consideration of the homogeneous form of (1) which is

$$
\left[\rho g W+\kappa-\omega^{2}\{M+a(\omega)+\mathrm{i}(b(\omega)+\gamma) / \omega\}\right] v=0
$$

It follows that necessary conditions for the existence of a non-zero $v$ (so that the structure is in motion) for some particular frequency $\omega=\omega_{0}$ are that

$$
\rho g W+\kappa-\omega_{0}^{2}\left\{M+a\left(\omega_{0}\right)\right\}=0
$$


and

$$
b\left(\omega_{0}\right)+\gamma=0 .
$$

For the fluid motion to have finite energy there can be no radiation of waves to infinity and hence, at the frequency $\omega_{0}$, it is required that the damping coefficient $b$ is zero. It then follows from (4) that the mooring characteristic $\gamma$ must also be zero. The resonance condition (3) can, in principle, be satisfied at any frequency by an appropriate choice of the spring constant $\kappa$ (although in some cases $\kappa$ would be negative). The focus here is on structures without moorings, and in this case the construction of a freely-floating trapping structure requires that both

$$
\rho g W-\omega_{0}^{2}\left\{M+a\left(\omega_{0}\right)\right\}=0
$$

and

$$
b\left(\omega_{0}\right)=0 .
$$

So-called wave-free structures, for which $b\left(\omega_{0}\right)=0$, have been studied by a number of authors. For example, Kyozuka \& Yoshida (1981) obtain such structures by an inverse procedure in which the velocity potential for the fluid motion is expressed in terms of singular solutions of the governing equations, and then the surface of a structure is obtained from examination of the streamlines of a modified flow. In general, the structure so obtained will not possess hydrodynamic characteristics that also allow equation (5) to be satisfied at the same frequency $\omega=\omega_{0}$. However, insight on how this might be achieved may be obtained through an application of Green's theorem as follows.

The result will be explained in detail for two-dimensional motion in water of infinite depth. Denote by $\phi_{0}$ the velocity potential corresponding to the vertical oscillations of a wave-free structure at frequency $\omega=\omega_{0}$ so that, in particular,

$$
\frac{\partial \phi_{0}}{\partial n}=n_{z} \quad \text { on } \quad \Gamma
$$

where $n$ is a normal coordinate to the wetted surface of the structure $\Gamma$, directed out of the fluid, and $n_{z}$ is the vertical component of the unit normal to $\Gamma$. By definition, the added mass

$$
a\left(\omega_{0}\right)=\rho \int_{\Gamma} \phi_{0} n_{z} \mathrm{~d} s
$$

where the fact that $b\left(\omega_{0}\right)=0$ has been used. With the assumption of symmetry about $x=0$, any wave-free potential must satisfy

$$
\phi_{0}=\frac{\mu \cos \theta}{R}+o\left(\frac{1}{R}\right) \quad \text { as } \quad R \rightarrow \infty,
$$

where $\mu$ is a constant, so that to leading order $\phi_{0}$ is dipole-like at infinity (Ursell, 1950); it is possible that $\mu$ may be zero.

Let $S$ denote the union of $\Gamma$ with the free surface $F$ and a closing semicircle $S_{\infty}$ at infinity in $z<0$. Application of Green's theorem over $S$ to the harmonic functions $\phi_{0}$ and

$$
u=z+1 / K
$$


yields

$$
\int_{S}\left[\phi_{0} \frac{\partial u}{\partial n}-u \frac{\partial \phi_{0}}{\partial n}\right] \mathrm{d} s=0
$$

The contribution to the integral from $F$ is zero, as both $\phi_{0}$ and $u$ satisfy the free-surface condition, and it follows from the asymptotic form (9) that

$$
\int_{S_{\infty}}\left[\phi_{0} \frac{\partial u}{\partial n}-u \frac{\partial \phi_{0}}{\partial n}\right] \mathrm{d} s=-\pi \mu .
$$

From the boundary condition (7),

$$
\int_{\Gamma}\left[\phi_{0} \frac{\partial u}{\partial n}-u \frac{\partial \phi_{0}}{\partial n}\right] \mathrm{d} s=\int_{\Gamma}\left[\phi_{0} n_{z}-\left(z+\frac{1}{K}\right) n_{z}\right] \mathrm{d} s .
$$

The integral of $\phi_{0}$ is written in terms of the added mass using (8). The divergence theorem may be used to show that for an arbitrary $\chi$

$$
\int_{\Gamma+W} \chi n_{z} \mathrm{~d} s=-\iint_{V} \nabla \chi \cdot \mathbf{e}_{z} \mathrm{~d} V
$$

where $V(=M / \rho)$ is the submerged volume of the structure (per unit length in the $y$ direction), $\mathbf{e}_{z}$ is a unit vector in the $z$ direction, and the normal coordinate $n$ is directed into $V$. The choice $\chi=z$ then gives

$$
\int_{\Gamma} z n_{z} \mathrm{~d} s\left(=\int_{\Gamma} z \mathrm{~d} x\right)=-V
$$

and the choice $\chi=1$ yields

$$
\int_{\Gamma} n_{z} \mathrm{~d} s=-\int_{W} n_{z} \mathrm{~d} s=\int_{W} \mathrm{~d} x=W
$$

Thus, the application of Green's theorem results in

$$
\rho g W-\omega_{0}^{2}\left\{M+a\left(\omega_{0}\right)\right\}=-\pi \mu \rho \omega_{0}^{2}
$$

and it is apparent that the resonance condition (5) can be satisfied if and only if the dipole coefficient $\mu$ is zero.

For two-dimensional motion in fluid of constant finite depth $h$ a similar application of Green's theorem leads to

$$
\rho g W-\omega_{0}^{2}\left\{M+a\left(\omega_{0}\right)\right\}=-\left.\rho \omega_{0}^{2} \int_{-\infty}^{\infty} \phi_{0}\right|_{z=-h} \mathrm{~d} x
$$

so that, given a wave-free potential $\phi_{0}$ at a frequency $\omega=\omega_{0}$, a necessary condition for the existence of a motion trapped mode at that frequency is

$$
\left.\int_{-\infty}^{\infty} \phi_{0}\right|_{z=-h} \mathrm{~d} x=0 .
$$




\section{Construction of motion trapping structures}

Motion trapping structures may be constructed using a modification of the inverse procedure described by Kyozuka \& Yoshida (1981). Let $\phi_{0}$ be a wave-free potential with dipole coefficient $\mu=0$; typically $\phi_{0}$ will be singular at a number of discrete points in $z \leq 0$. From equation (7), if a suitable structure could be identified, the modified potential

$$
\phi=z-\phi_{0}
$$

would satisfy

$$
\frac{\partial \phi}{\partial n}=0 \quad \text { on } \quad \Gamma
$$

so that $\Gamma$ is a streamline of the flow corresponding to $\phi$. Hence motion trapping structures are obtained from any streamlines of this flow that isolate the singularities of $\phi$ from infinity.

The condition (21) can be satisfied if the near field to the structure contains a dipole component, while the resonance condition (5) holds only if there is no dipole component in the far field. This can be arranged using the following construction that uses a wave-free potential for the fluid motion of the trapped mode that has two components. The first component is

$$
\phi_{\mathrm{s}}=f_{0}^{\infty} \frac{\mathrm{e}^{\mu z} \cos \mu(x-\xi)}{\mu-K} \mathrm{~d} \mu+f_{0}^{\infty} \frac{\mathrm{e}^{\mu z} \cos \mu(x+\xi)}{\mu-K} \mathrm{~d} \mu
$$

and is a combination of logarithmically singular wave sources in the free surface at $x= \pm \xi$ that satisfies Laplace's equation, the free-surface condition and, provided that $K \xi=(2 n+1) \pi / 2$ for some integer $n \geq 0$, is wave-free at infinity (for all of the calculations reported here $n$ is chosen to be zero). This potential was used by McIver (1996) in the construction of sloshing trapped modes for fixed structures. The potential $\phi_{\mathrm{s}}$ may be written in terms of the exponential integral $\mathrm{E}_{1}$ as

$$
\begin{aligned}
\phi_{\mathrm{S}}=(-1)^{n} \operatorname{Re}\left\{\mathrm{e}^{K z+\mathrm{i} K x}[\pi \operatorname{sgn}(x-\xi)-\right. & \pi \operatorname{sgn}(x+\xi) \\
& \left.\left.-\mathrm{i} \mathrm{E}_{1}(K z+\mathrm{i} K(x-\xi))+\mathrm{i} \mathrm{E}_{1}(K z+\mathrm{i} K(x+\xi))\right]\right\}
\end{aligned}
$$

and the corresponding stream function is

$$
\begin{aligned}
\psi_{\mathrm{s}}=(-1)^{(n+1)} \operatorname{Im}\left\{\mathrm{e}^{K z+\mathrm{i} K x}[\pi \operatorname{sgn}(x-\xi)\right. & -\pi \operatorname{sgn}(x+\xi) \\
& \left.\left.-\mathrm{i}_{1}(K z+\mathrm{i} K(x-\xi))+\mathrm{i} \mathrm{E}_{1}(K z+\mathrm{i} K(x+\xi))\right]\right\}
\end{aligned}
$$

From equation (23) and standard asymptotic results (Abramowitz \& Stegun, 1964, equation 5.1.51) it may be shown that

$$
\phi_{\mathrm{s}} \sim-\frac{2 \cos \theta}{K R} \quad \text { as } \quad R=\left(x^{2}+z^{2}\right)^{1 / 2} \rightarrow \infty .
$$

Thus $\phi_{\mathrm{S}}$ has the property that it is logarithmic near to $(x, z)=( \pm \xi, 0)$ but dipole-like in the far field. The second component of the wave-free potential is

$$
\phi_{\mathrm{d}}=\frac{1}{K^{2}}\left[\phi_{1}\left(R_{+}, \theta_{+}\right)+\phi_{1}\left(R_{-}, \theta_{-}\right)\right]
$$




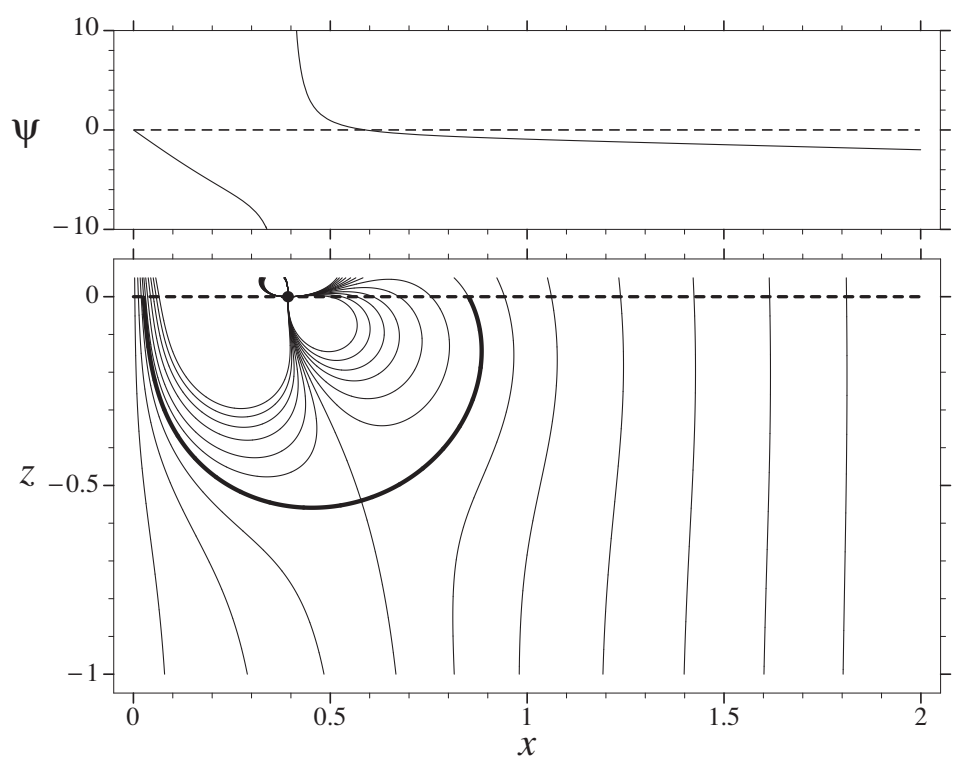

Figure 1: The lower figure shows the streamlines corresponding to the stream function (31) and the upper figure shows the same stream function plotted on $z=0 ; K=4 ; n=0, \delta=1$.

where $R_{ \pm}, \theta_{ \pm}$are polar coordinates with origin at $(x, z)=( \pm \xi, 0)$ and

$$
\phi_{1}(R, \theta)=\frac{K \cos \theta}{R}+\frac{\cos 2 \theta}{R^{2}}
$$

is a standard wave-free potential that satisfies Laplace's equation and the free-surface condition; the stream function corresponding to $(28)$ is

$$
\psi_{1}(R, \theta)=-\frac{K \sin \theta}{R}-\frac{\sin 2 \theta}{R^{2}}
$$

The potential $\phi_{\mathrm{d}}$ is singular at the same points as $\phi_{\mathrm{s}}$, has a dipole component in the near field to each singularity, and to leading order is dipole-like in the far field so that

$$
\phi_{\mathrm{d}} \sim \frac{2 \cos \theta}{K R} \quad \text { as } \quad r \rightarrow \infty .
$$

With the above properties of $\phi_{\mathrm{s}}$ and $\phi_{\mathrm{d}}$ in mind, the wave-free potential to be employed in the construction of motion trapping structures is

$$
\phi_{0}=\delta\left(\phi_{\mathrm{s}}+\phi_{\mathrm{d}}\right)
$$

where $\delta$ is an arbitrary positive constant (it is demonstrated below in $\S 4.1$ that suitable stream lines can be found for any $\delta>0)$. This choice for $\phi_{0}$ has all of the required properties and, in particular, no dipole component in its far-field expansion. From Ursell (1950), it is known that in the far-field any potential symmetric about $x=0$ has an expansion in terms of a wave source and wave-free potentials. Thus, the elimination from $\phi_{\mathrm{s}}$ (which is already wave-free) of the dipole component using (28) also eliminates the quadrupole component so that $\phi_{0}=O\left(1 / R^{3}\right)$ as $R \rightarrow \infty$. 


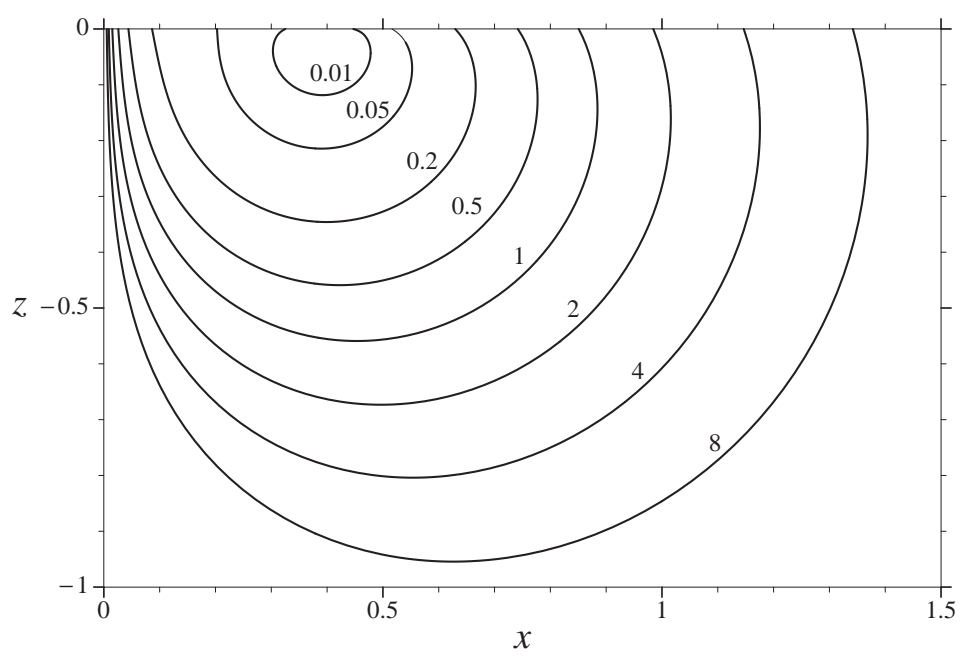

Figure 2: The right-hand element of trapping structures obtained from the stream function (31); $K=4, n=0$. Each structural surface is marked with the corresponding value of $\delta$.

Motion trapping structures are obtained by examination of the stream function

$$
\psi=-x-\delta\left(\psi_{\mathrm{s}}+\psi_{\mathrm{d}}\right)
$$

where

$$
\psi_{\mathrm{d}}=\frac{1}{K^{2}}\left[\psi_{1}\left(R_{+}, \theta_{+}\right)+\psi_{1}\left(R_{-}, \theta_{-}\right)\right]
$$

The lower part of figure 1 shows the streamlines of $\psi$ in $x>0$ for the case $K=4, \delta=1$. (The wavenumber $K$ could be scaled out of the problem, but it is convenient not to do this in view of some of the computations for finite depth presented later in the paper.) The streamline pattern for $x<0$ is obtained by reflection in $x=0$. This figure is typical in that, for $x>0$, there is a single dividing streamline part of which (shown by a thick line in the figure) forms a contour that, in the fluid region, isolates the singular point from infinity; the part of this contour that lies in $z<0$, together with its reflection in $x=0$, forms the wetted surface $\Gamma$ of the motion trapping structure. It should be born in mind that the streamline pattern shown in Figure 1 does not correspond to that in the water-wave problem, it serves only to determine the contour $\Gamma$. The value of the stream function on the dividing streamline is obtained by numerically locating the stagnation point of the flow and inserting the coordinates into the stream function. Once the dividing streamline is known the resonance condition (5) can be verified by computing the added mass from equation (8) and the submerged volume (and hence the mass) from equation (15).

Although for each $K$ and $\delta$ the potential $\phi_{0}$ generates a single motion trapping structure, continuous variation of $\delta$ gives a range of structures with the same resonant wave number $K$. Numerical experimentation suggests that trapping structures can only be obtained for $\delta>0$ and some of the structures obtained for $K=4$ are shown in figure 2. For most of the values of $\delta$ illustrated, the structures resemble the sloshing trapping structures obtained by McIver (1996) in 
that the so-called John condition is satisfied on the inside of the structural element (small $x$ ), so that vertical lines drawn down from the free surface do not intersect $\Gamma$, but on the outside of the element the John condition is violated. However, for small $\delta$ the John condition is violated on both sides of the element (Motygin \& Kuznetsov, 1998, obtained a family of sloshing trapping structures that violate the John condition on both sides of an element).

\subsection{The streamline pattern}

In this section the streamline pattern associated with the potential $\phi=z-\phi_{0}$ is discussed and, in particular, it is shown that motion trapping structures are possible for any positive value of the parameter $\delta$ that appears in equation (30). The stream function $\psi$ associated with $\phi$ is

$$
\begin{aligned}
\psi= & -x+\delta\left[f_{0}^{\infty} \frac{\mathrm{e}^{\mu z} \sin \mu(x-\xi)}{\mu-K} \mathrm{~d} \mu+f_{0}^{\infty} \frac{\mathrm{e}^{\mu z} \sin \mu(x+\xi)}{\mu-K} \mathrm{~d} \mu\right. \\
& \left.+\frac{(x+\xi)}{K\left[z^{2}+(x+\xi)^{2}\right]}-\frac{2 z(x+\xi)}{K^{2}\left[z^{2}+(x+\xi)^{2}\right]^{2}}+\frac{(x-\xi)}{K\left[z^{2}+(x-\xi)^{2}\right]}-\frac{2 z(x-\xi)}{K^{2}\left[z^{2}+(x-\xi)^{2}\right]^{2}}\right] .
\end{aligned}
$$

This function is antisymmetric in $x$ so that $\psi(0, z)=0$, and to leading order $\psi$ has a quadrupole singularity at $(x, z)=(\xi, 0)$. From an investigation of the variation of the stream function along $z=0$, we will demonstrate that if $\delta>0$ and $K \xi=\pi / 2$, there is a streamline of the function which leaves the free surface from a point to the right of $x=\xi$ and re-enters it in the region $0<x<\xi$. By symmetry there is then a corresponding streamline in $x<0$, and this pair of streamlines form a motion trapping structure (in the following it is assumed that $x \geq 0$ ).

From (33) $\psi(x, 0)$ ranges from zero at $x=0$ to $-\infty$ as $x \rightarrow \xi^{-}$and $\psi(x, 0)$ ranges from $+\infty$ to $-\infty$ as $x$ varies from $\xi$ to $\infty$ (see the upper part of figure 1). From equation (33)

$$
\begin{aligned}
\frac{\partial \psi}{\partial x}(x, 0)= & -1 \\
& +K \delta\left[f_{0}^{\infty} \frac{\cos \mu(x-\xi)}{\mu-K} \mathrm{~d} \mu+f_{0}^{\infty} \frac{\cos \mu(x+\xi)}{\mu-K} \mathrm{~d} \mu-\frac{1}{K^{2}(x+\xi)^{2}}-\frac{1}{K^{2}(x-\xi)^{2}}\right] .
\end{aligned}
$$

The integrals in (34) may be rewritten with the use of contour integration and the quadrupole terms may also be expressed as integrals to give

$$
\begin{aligned}
\frac{\partial \psi}{\partial x}(x, 0)=-1+K \delta & {\left[-\pi(\sin K|x-\xi|+\sin K(x+\xi))+\int_{0}^{\infty} \frac{\mu \mathrm{e}^{-\mu|x-\xi|}}{K^{2}+\mu^{2}} \mathrm{~d} \mu+\int_{0}^{\infty} \frac{\mu \mathrm{e}^{-\mu(x+\xi)}}{K^{2}+\mu^{2}} \mathrm{~d} \mu\right.} \\
& \left.-\frac{1}{K^{2}} \int_{0}^{\infty} \mu \mathrm{e}^{-\mu|x-\xi|} \mathrm{d} \mu-\frac{1}{K^{2}} \int_{0}^{\infty} \mu \mathrm{e}^{-\mu(x+\xi)} \mathrm{d} \mu\right] \\
=-1- & K \delta[\pi(\sin K|x-\xi|+\sin K(x+\xi)) \\
+ & \left.\frac{1}{K^{2}} \int_{0}^{\infty} \frac{\mu^{3} \mathrm{e}^{-\mu|x-\xi|}}{K^{2}+\mu^{2}} \mathrm{~d} \mu+\frac{1}{K^{2}} \int_{0}^{\infty} \frac{\mu^{3} \mathrm{e}^{-\mu(x+\xi)}}{K^{2}+\mu^{2}} \mathrm{~d} \mu\right]
\end{aligned}
$$

Thus if $K \xi=\pi / 2$

$$
\frac{\partial \psi}{\partial x}(x, 0)=-1-\frac{\delta}{K}\left[\int_{0}^{\infty} \frac{\mu^{3} \mathrm{e}^{-\mu|x-\xi|}}{K^{2}+\mu^{2}} \mathrm{~d} \mu+\int_{0}^{\infty} \frac{\mu^{3} \mathrm{e}^{-\mu(x+\xi)}}{K^{2}+\mu^{2}} \mathrm{~d} \mu\right], \quad \text { for } \quad x>\xi,
$$


and for $0 \leq x<\xi$

$$
\frac{\partial \psi}{\partial x}(x, 0)=-1-\frac{\delta}{K}\left[2 \pi \cos K x+\int_{0}^{\infty} \frac{\mu^{3} \mathrm{e}^{-\mu|x-\xi|}}{K^{2}+\mu^{2}} \mathrm{~d} \mu+\int_{0}^{\infty} \frac{\mu^{3} \mathrm{e}^{-\mu(x+\xi)}}{K^{2}+\mu^{2}} \mathrm{~d} \mu\right] .
$$

As $0 \leq K x<\pi / 2$ in (37), $\partial \psi / \partial x(x, 0)<0 \forall x \neq \xi$, and so $\psi$ monotonically decreases from $+\infty$ to $-\infty$ as $x$ varies from $\xi$ to $\infty$ and $\psi$ monotonically decreases from 0 to $-\infty$ as $x$ varies from 0 to $\xi$.

A streamline which emanates from the free surface to the right of the singular point, on which $\psi>0$, cannot cross the $z$ axis, as $\psi=0$ there. In addition it cannot go off to infinity in $x>0$ as $\psi \sim-x$ as $x^{2}+z^{2} \rightarrow \infty$, nor can it return to the free surface as there is no other place at which $\psi>0$ than where it started. Thus the streamline must terminate at the singular point, which is possible as the flow field is quadrupole like in the immediate neighbourhood of that point.

At large distances to the right of the singular point the streamlines asymptote to those for a uniform stream in the $z$ direction, so streamlines coming out of the free surface for large values of $x$ go to negative infinity in $z$. Thus there is a dividing streamline which emanates from the free surface to the right of the singular point, on which $\psi=\psi_{\mathrm{d}} \leq 0$, and which separates the streamlines that enter the singular point from those which tend to infinity. If $\psi_{\mathrm{d}}=0$ then the dividing streamline must connect to the $x$ axis rather than the free surface and so form a structure with one element rather than two, but in practice this does not seem to happen.

Assume now that $\psi_{\mathrm{d}}<0$. If there is no stagnation point on the dividing streamline in $z<0$ then it must meet the free surface at some $x$ in the interval $(0, \xi)$ and hence form one element of a two-element structure. However this situation is impossible as the resulting flow pattern would not be able to accommodate those streamlines that tend to negative infinity in $z$ and for which $\psi \in\left(-\infty, \psi_{\mathrm{d}}\right)$. If the dividing streamline does come to a stagnation point (of any order) in $z<0$ then one branch of the streamline that emerges from the stagnation point must go to infinity, and at least one branch must go into the singularity. If there is no other branch with $\psi=\psi_{\mathrm{d}}$, then the streamlines that emerge from the free surface with $\psi<\psi_{\mathrm{d}}$ could not be accommodated into the flow pattern as streamlines at large depths with this range of stream function values already exist to the right of the dividing stream line. Hence one branch of the streamline from the stagnation point must join the free surface to the left of the singular point, where it divides streamlines emanating from this portion of the free surface into those entering the singular point and those going to negative infinity in $z$. (If there are further stagnation points then the above arguments can be repeated with minor modifications, although numerical calculations suggest that there is a single stagnation point.) The portion of the dividing streamline which connects to the free surface, plus its reflection in the $z$ axis, removes the singularities from the flow field and so may be taken to be the boundary of a motion trapping structure. Thus, for a given $K$ a trapping structure may be found for any $\delta>0$. 

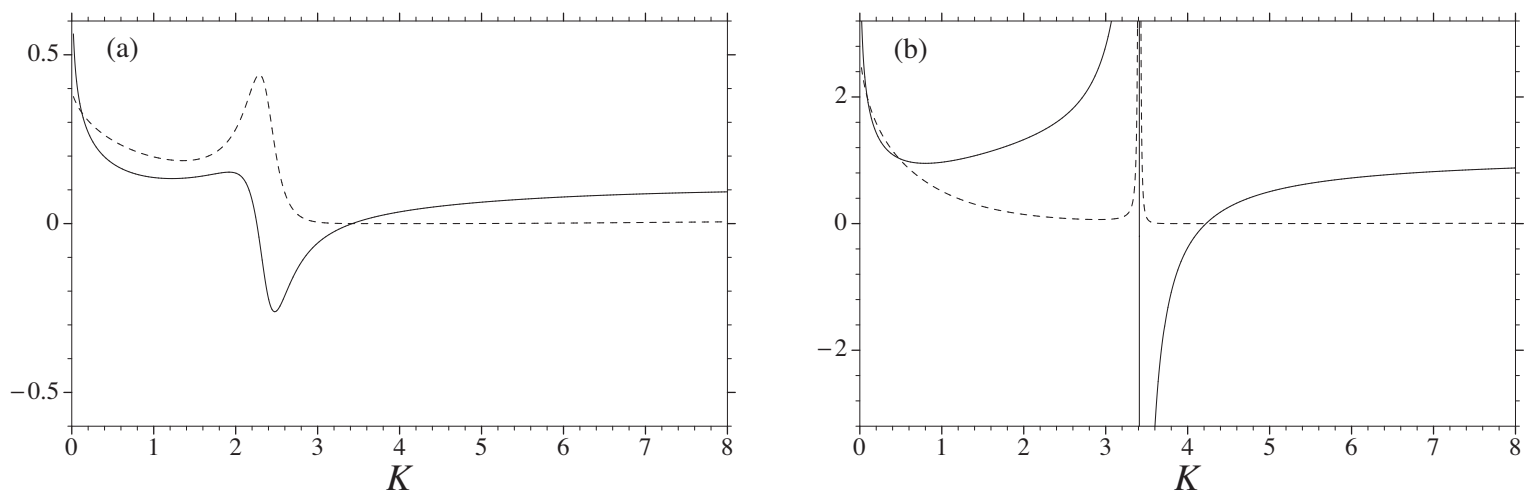

Figure 3: Heave added mass $a / \rho(-)$ and damping $b / \rho \omega\left(--{ }_{-}\right)$coefficients for motion trapping structures with $K=4$ and (a) $\delta=0.05$, (b) $\delta=1$.

\section{Consequences of the existence of motion trapped modes}

\subsection{The frequency domain}

In the case of a sloshing trapping structure, the frequency-domain radiation potential has a pole at the trapped-mode frequency, but the scattering potential is orthogonal to the trapped mode and hence is well-behaved at the trapped-mode frequency. Newman (1999) has performed detailed numerical calculations of the frequency-domain hydrodynamic coefficients for fixed trapping structures in three dimensions. The most striking manifestation of the singularity in the radiation potential is the very rapid change in the added mass coefficient that occurs around the trapped-mode frequency.

For a motion trapping structure the scattering and radiation potentials are both well behaved at the resonant frequency $\omega=\omega_{0}$. Consequently, the hydrodynamic coefficients for such a structure are analytic functions of the frequency $\omega$ for all real positive values. By construction the damping coefficient $b$ is zero at the resonant frequency $\omega=\omega_{0}$ and hence, from the Haskind relation, the exciting force $X\left(\omega_{0}\right)=0$. The added mass and damping coefficients for two motion trapping structures are shown in figure 3 (the corresponding structures are shown in figure 2). In both cases motion trapping occurs for $K=4$ and at lower frequencies there are rapid changes in the added mass and damping that are associated with a pole of the radiation potential in the lower half of the complex frequency plane. These poles correspond to sloshing complex resonances associated with fluid motions between the two elements of each structure (see McIver 2005). In case (a) the region of rapid change is well away from the motion trapped mode at $K=4$ where the added mass in positive. This contrasts with case (b) in which there are much stronger changes in added mass and damping and at $K=4$ the added mass is negative. In both cases the zero in the damping at $K=4$ occurs in a region where the damping is in any case very small.

Singular behaviour in a problem involving a motion trapping structure may arise through the solution of the equation of motion (1) for $v(\omega)$. If one or both of the initial displacement and velocity, $Z(0)$ and $\dot{Z}(0)$ respectively, are non zero then from (1) the velocity $v$ will have a simple 


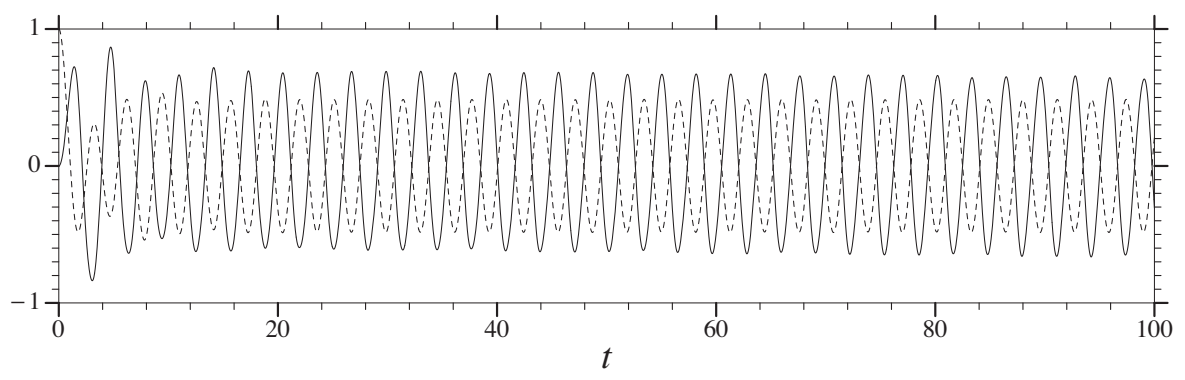

Figure 4: Displacement $\eta / h$ of the mid point of the internal free surface $(-)$ and displacement $\zeta / h$ of the structure $(---)$ due to release of the structure from rest.

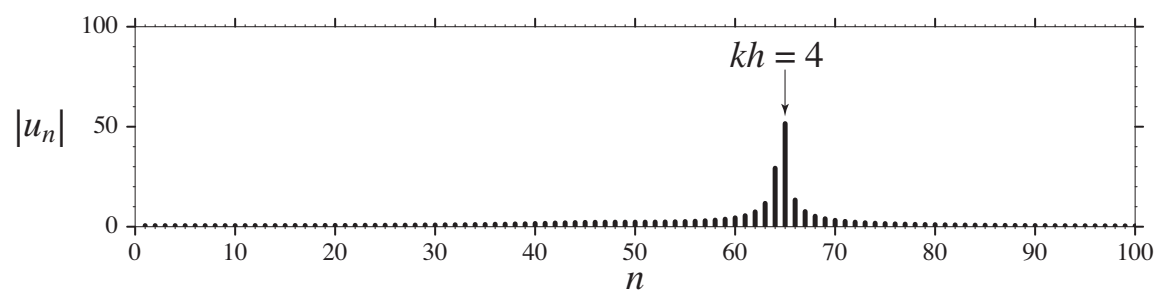

Figure 5: Fourier transform of the displacement, shown in figure 4, of the mid point of the internal free surface due to release of the structure from rest.

pole at $\omega=\omega_{0}$. Thus, in these circumstances, the solution to the boundary-value problem will not exist at the resonant frequency. However, if $Z(0)=\dot{Z}(0)=0$ then the analytic nature of $X(\omega)$ means that the zero in $X$ annuls the zero arising from (5) and (6) and so $v(\omega)$ is non-singular at the resonant frequency and the solution to the boundary-value problem exists, although it will not be unique.

\subsection{The time domain}

Any persistent oscillation in the time domain with frequency $\omega_{0}$ corresponds to a simple pole in the frequency-domain potential at a real frequency $\omega=\omega_{0}$ (see McIver 2005). In the discussion of $\S 5.1$ it was noted that for a motion trapping structure such poles will occur only as a result of a non-zero initial displacement and/or velocity. Thus, for example, a motion trapped mode will be excited in the time domain by displacing the corresponding trapping structure from its equilibrium position and releasing it from rest. This is illustrated in figure 4 by means of a numerical calculation made using the method described by McIver et al. (2003). This method is for two-dimensional motion in fluid of constant finite depth $h$ and hence a modification of the constructive procedure described in $\S 4$ is needed. For the structure used in the calculations $\phi_{\mathrm{S}}$ is taken to be a pair of wave sources and $\phi_{\mathrm{d}}$ a pair of wave dipoles (Yu \& Ursell 1961) with the spacing $2 \xi$ and finite-depth wave number $k$ chosen to satisfy $k \xi=\pi / 2$, where $k$ is related to $K$ through $K=k \tanh k h$. The particular 


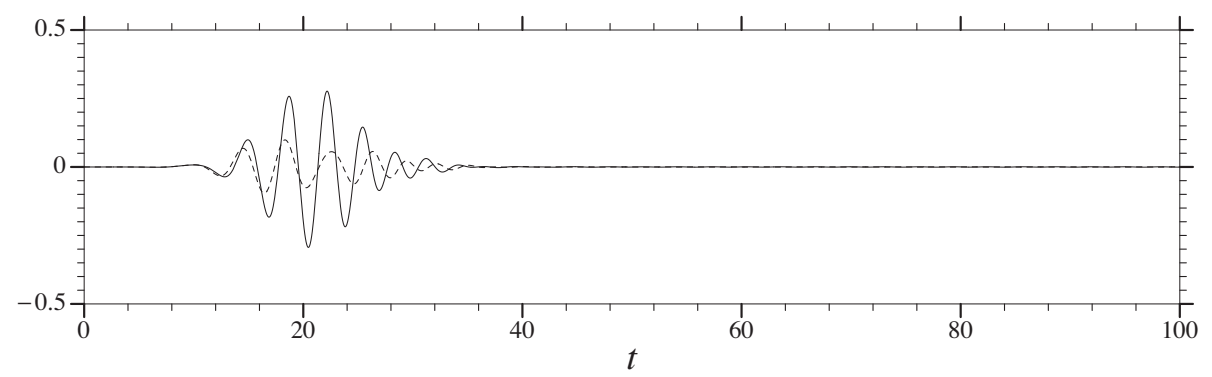

Figure 6: Displacement $\eta / h$ of the mid point of the internal free surface $(-)$ and displacement $\zeta / h$ of the structure $(---)$ due to an incident wave packet.

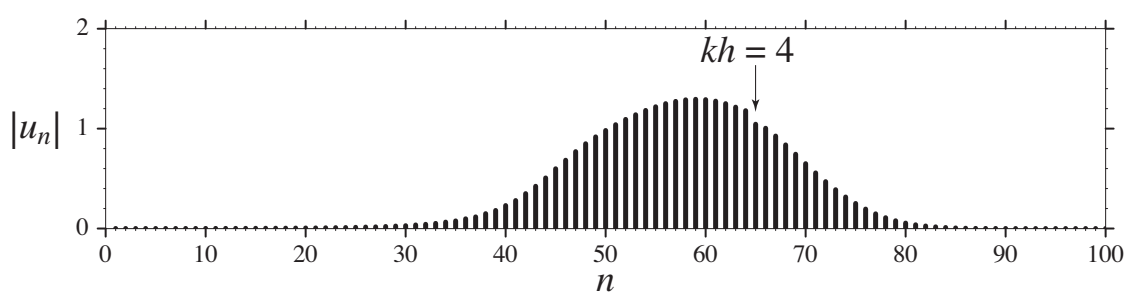

Figure 7: Fourier transform of the displacement, shown in figure 6, of the mid point of the internal free surface due to an incident wave packet .

trapping structure used here has a trapped-mode frequency corresponding to $k h=4$ and the parameter $\delta=0.05$ (the shape of the structure obtained is very close to that shown in figure 2 for infinite depth with $K=4$ and $\delta=0.05$ ). Figure 4 shows the displacement of the structure and the free-surface elevation at the mid point of the internal free surface. After an initial transient has decayed both motions settle to a steady oscillation with the same frequency. The discrete Fourier transform of the surface elevation shown in figure 5 confirms that this oscillation is at the trapped-mode frequency (see McIver et al. 2003 for further details of the transform).

If a motion trapping structure is initially at rest in its equilibrium position then, as explained in $\S 5.1$, the frequency-domain velocity $v(\omega)$ does not have a pole at the resonant frequency. Consequently if a time-domain motion is excited by an incident wave there can be no persistent oscillation. This is illustrated in figure 6 for the same structure used for the calculation shown in figure 4 . The structure is set in motion by a incident Gaussian wave packet with a peak frequency corresponding to $k h=4$ (see equation (31) of McIver 2005 for the definition of the wave packet used). After interaction with the incident wave the motion of the internal free surface and the structure both rapidly die away and the Fourier transform of the free-surface confirms the absence of a persistent oscillation at the resonant frequency. 


\section{Other modes of motion}

This paper has concentrated on heaving motion trapping structures, but it is likely that similar constructions are possible for other modes of motion. This is demonstrated here for two-dimensional horizontal motions in water of infinite depth. For such surge motions the hydrostatic restoring force does not appear in the equation of motion and the construction of a motion trapping structure at a frequency $\omega=\omega_{0}$ requires that the surge added mass coefficient $a(\omega)$ satisfies

$$
M+a\left(\omega_{0}\right)=0
$$

and that the surge damping coefficient is zero. Equation (38) indicates that the added mass is negative at a trapped-mode frequency $\omega=\omega_{0}$ which suggests that, in this case, the motion trapped mode is closely associated with a pole of the radiation potential in the lower half of the complex frequency plane that lies close to the real frequency axis near $\omega=\omega_{0}$. With the assumption of geometrical symmetry about $x=0$, a wave-free potential corresponding to a horizontal oscillation about $x=0$ has the form

$$
\phi_{0}=\frac{\mu \sin 2 \theta}{R^{2}}+o\left(\frac{1}{R^{2}}\right) \quad \text { as } \quad R \rightarrow \infty
$$

(Ursell 1950). An application of Green's theorem to $\phi_{0}$ and $u=x$ similar to that described for heave in $\S 3$ shows that the resonance condition (38) is satisfied if and only if

$$
\int_{F} x \phi_{0} \mathrm{~d} x=0
$$

where $F$ is the free surface. The required boundary condition,

$$
\frac{\partial \phi_{0}}{\partial n}=n_{x} \quad \text { on } \quad \Gamma
$$

can be satisfied by examination of the streamlines of the potential

$$
\phi=x-\phi_{0}
$$

where

$$
\phi_{0}=\delta \frac{\partial \phi_{\mathrm{s}}}{\partial x}
$$

and $\phi_{s}$ is the combination of wave sources given in equation (22) (and hence $\phi_{0}$ is formed from a pair of horizontal wave dipoles).

Here the choices $K=1, n=0$ and $\delta=20$ are made and the corresponding streamline pattern is shown in figure 8 for $x \geq 0$. To construct a motion trapping structure a streamline is first chosen from those that surround the singular point in $z \leq 0$. A second streamline is then chosen from those that intersect $x=0$ to ensure that equation (40) is satisfied. (This is possible because the potential $\phi_{0}$ changes sign across $x= \pm \xi$.) One combination of streamlines which, together with their reflections in $x=0$, are able to form a motion trapping structure is shown using thicker lines in figure 8. The complete structure has three elements and is typical of many calculations (for 


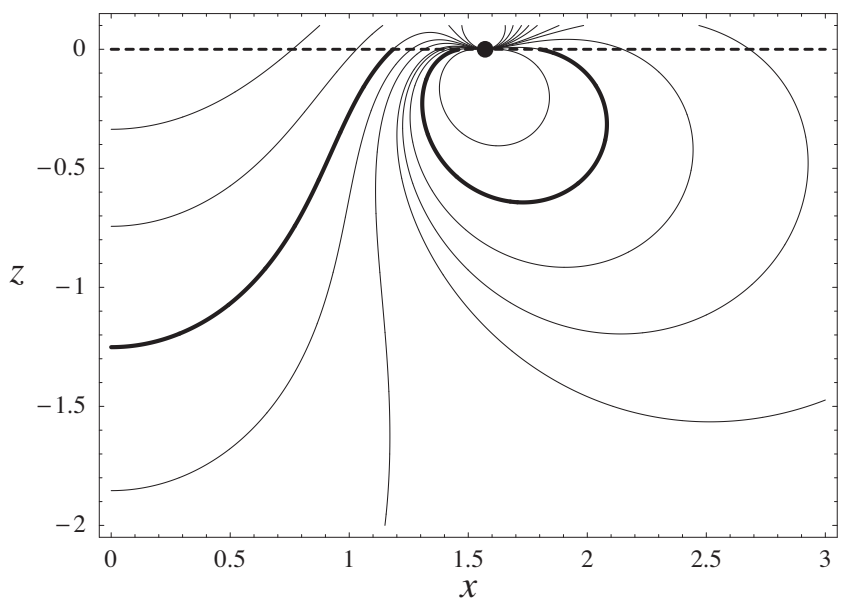

Figure 8: Streamlines corresponding to the potential $x-\phi_{0}$ with $\phi_{0}$ given by equation (43), and with $K=1$ and $\delta=20$. The singular point is indicated by a filled disk.
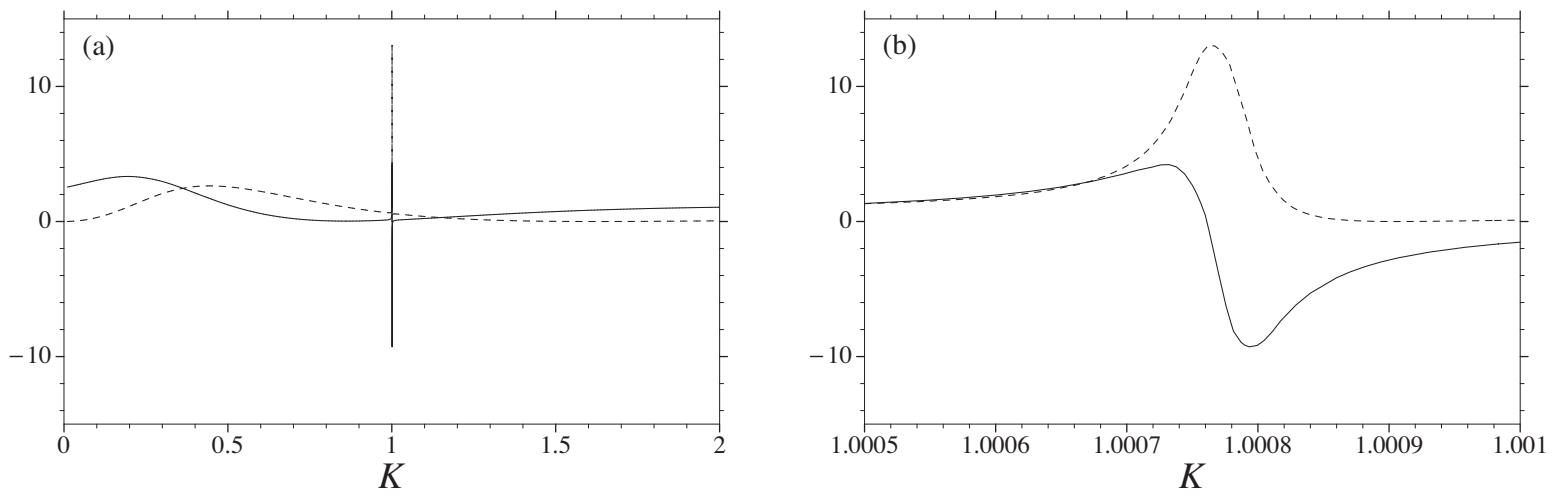

Figure 9: Surge added mass $a / \rho(-)$ and damping $b / \rho \omega\left(---_{-}\right)$coefficients for the motion trapping structure shown in figure 8; a motion trapped mode exists at $K=1$.

different values of $\delta$ and choices of streamline around the singularity) in that the central element has a significantly larger draft than the outer elements, and the gaps between elements are relatively narrow compared to the overall dimensions of the structure. In contrast to the heave case discussed in $\S 4$, one streamline pattern yields a range of trapping structures.

The added mass and damping coefficients calculated by a panel method are given in figure 9 for the motion trapping structure shown in figure 8. The motion trapping is closely associated with a very narrow banded resonance around $K=1$ that could easily be overlooked in a numerical investigation of the hydrodynamic properties of this structure. This contrasts with the particular heave motion trapping structures discussed earlier for which the frequency of the motion resonance was well separated from the most rapid changes in the added mass and damping coefficients. The streamline pattern in figure 8 suggests that the resonance in the radiation problem corresponds 
to out of phase pumping oscillations in the two gaps between structural elements (although the constructed streamlines correspond to the motion resonance at $K=1$ ). A blow up of the resonant region is given in figure 9 (b). Due to the numerical error in the results of the panel method there is a frequency shift and the minimum in the damping actually occurs at $K=1.000907$ rather than the value $K=1$ used in the original construction. Further indication of the level of numerical error is given by the fact that at $K=1.000907$ the added mass calculated by the panel method is -2.69 rather than the value -2.78 calculated directly from the exact velocity potential $\phi_{0}$ with $K=1$.

\section{Conclusion}

It has been demonstrated that, within the linearized theory of water waves, there are freely-floating structures that support trapped modes. Such modes are free oscillations involving coupled motions of the structure and fluid that do not radiate waves to infinity and which, in the absence of viscosity, will persist for all time. The structures that support such modes are termed here motion trapping structures and can be contrasted with the previously-known sloshing trapping structures that, when held fixed, can support free oscillations of the fluid. Examples of motion trapping structures have been constructed for both heave and surge motions in two dimensions. Examination of the equations of motion shows that motion trapped modes cannot be excited by incident waves, but they can be excited by giving the structure a non-zero initial displacement and/or velocity. This has been confirmed by numerical calculations in the time domain.

\section{References}

Abramowitz, M. \& Stegun, I. A. 1964 Handbook of Mathematical Functions. National Bureau of Standards, Washington.

John F. 1950 On the motion of floating bodies II. Comm. Pure Appl. Maths, 3, 45-101.

Kuznetsov N. 2003 On the motion of a floating body supported by an air cushion. Mathematical and Numerical Aspects of Wave Propagation: WAVES 2003, 483-488. Springer.

Kyozuka, Y \& Yoshida, K. 1981 On wave-free floating body forms in heaving oscillation. Appl. Ocean Res., 3, 183-194.

McIver, M. 1996 An example of non-uniqueness in the two-dimensional linear water wave problem. J. Fluid Mech., 315, 257-266.

McIver, M. 1997 Resonances in the unbounded water wave problem. Proc. 12th International Workshop on Water Waves and Floating Bodies, held in Carret-le-Rouet, France, 16-19 March 1997, 177-180.

McIver, P. 2005 Complex resonances in the water-wave problem for a floating structure. J. Fluid Mech., 536, 423-443. 
McIver, P. \& McIver, M. 1997 Trapped modes in an axisymmetric water-wave problem. Q. J. Mech. Appl. Math., 50, 165-178.

McIver, P., McIver, M. \& Zhang, J. 2003 Excitation of trapped modes by the forced motion of structures. J. Fluid Mech., 494, 141-162.

Motygin O. \& Kuznetsov, N.. 1998 Non-uniqueness in the water-wave problem: an example violating the inside John condition. Proc. 12th International Workshop on Water Waves and Floating Bodies, held in Alphen aan den Rijn, The Netherlands, 29 March - 1 April 1998, $107-110$.

Newman, J. N. 1999 Radiation and diffraction analysis of the McIver toroid. J. Eng. Math., 35, $135-147$.

Ursell, F. 1950 Surface waves on deep water in the presence of a submerged circular cylinder. II Proc. Camb. Phil. Soc., 46, 153-158.

Yu, Y. S. \& Ursell, F. 1961 Surface waves generated by an oscillating circular cylinder on water of finite depth: theory and experiment. J. Fluid Mech., 11, 529-551. 